\title{
Effects of Dopamine Depletion on Visual Sensitivity of Zebrafish
}

\author{
Lei Li and John E. Dowling \\ Department of Molecular and Cellular Biology, Harvard University, Cambridge, Massachusetts 02138
}

\begin{abstract}
The visual sensitivity of zebrafish in which the retinal dopaminergic interplexiform cells (DA-IPCs) were destroyed by 6-hydroxydopamine was measured behaviorally. During the first 6-8 min of dark adaptation, visual thresholds of DA-IPC-depleted animals were similar to those of control animals. Thereafter, their visual thresholds were elevated so that by 14-18 min of dark adaptation, they were 2-3 log units above those of control animals. In DA-IPC-depleted animals, the electroretinogram was normal in terms of light sensitivity and waveform, but the light threshold for eliciting a ganglion cell discharge was raised by 1.8 log units as compared with control animals. No obvious rod system function was detected in DA-IPC-depleted animals as
\end{abstract}

measured behaviorally. Partial rescue of the behavioral visual sensitivity loss in DA-IPC-depleted animals occurred when dopamine or a long-acting dopamine agonist (2-amino-6, 7-dihydroxy-1, 2, 3, 4-tetrahydronaphthalene hydrobromide) were injected intraocularly. Our data suggest that the principal visual defect shown by DA-IPC-depleted animals is attributable to effects occurring in the inner retina, mainly on rod signals. We also show that dopamine is involved in mediating the effect of the circadian clock on visual sensitivity.

Key words: 6-OHDA; circadian clock; dark adaptation; dopamine; dopamine receptor agonist; ADTN; ERG recording; RGC recording; visual sensitivity; zebrafish
Dopamine plays an important modulatory role in the vertebrate visual system and has been shown to have both direct and indirect effects on every retinal neuron. Direct dopamine effects on retinal neurons include modulation of rod-cone photoreceptor coupling in frog (Krizaj et al., 1998), altered electrical coupling and glutamate receptor sensitivity of horizontal cells in fish (Teranishi et al., 1983; Knapp et al., 1990), modulation of glutamate-gated ionic currents in bipolar cells of tiger salamander (Maguire and Werblin, 1994), modification of gap junctional permeability between amacrine cells in rabbit (Hampson et al., 1992), and regulation of $\mathrm{Ca}^{2+}$ currents in ganglion cells of turtle (Liu and Lasater, 1994). The general view advanced from the single cell studies is that dopamine serves as a light signal in the retina, acting in particular to alter rod-cone input to second order cells (Witkovsky and Dearry, 1991). This conclusion has been arrived at by studies performed mainly in the outer retina and on horizontal cells.

In fish retinas, dopamine is released exclusively by dopaminergic interplexiform cells (DA-IPCs) (Dowling and Ehinger, 1975, 1978; Dowling, 1991). The DA-IPCs receive most of their synaptic input in the inner plexiform layer, mainly from amacrine cells and centrifugal fibers, and they make synapses in both the inner and outer plexiform layers (Zucker and Dowling, 1987; Yazulla and Zucker, 1988). It is generally accepted that light, especially flickering light, enhances dopamine release in the retina (Kramer, 1971; Bauer et al., 1980; Dearry and Burnside, 1989; Kirsch and Wagner, 1989; Umino and Dowling, 1991). However, evidence for a prolonged darkness-induced dopamine release also exists (Mangel and Dowling, 1985; Yang et al., 1988a,b; Xin and Bloomfield, 1999). In isolated perch retinas kept in darkness

\footnotetext{
Received Oct. 5, 1999; revised Dec. 16, 1999; accepted Dec. 17, 1999.

This work was supported by National Institutes of Health Grants EY 00811 and EY 00824. We thank A. Adolph for expert advice on ERG and ganglion cell recordings. We also thank E. Schmitt for sharing unpublished data, J. Fadool for providing 5E11 antibodies, and W. McCarthy, S. Harris, and S. Sciascia for maintenance of zebrafish.

Correspondence should be addressed to Lei Li, Department of Physiology, University of Kentucky College of Medicine, 800 Rose Street, Lexington, KY 40536. Copyright (C) 2000 Society for Neuroscience $0270-6474 / 00 / 201893-11 \$ 15.00 / 0$
}

for $2 \mathrm{hr}$, for example, the level of dopamine was elevated twofold (Weiler et al., 1997). The prolonged darkness-induced dopamine release in the perch retina was observed only at night, suggesting that the dopamine release is also modified by an endogenous circadian oscillator.

Here we report evidence for dopamine modulation of visual sensitivity using behavioral and electrophysiological methods in control and DA-IPC-depleted zebrafish. During the course of dark adaptation after bright light adaptation, visual thresholds of DA-IPC-depleted fish measured behaviorally were similar to those of control fish only for the first 6-8 min; thereafter, they increased and stabilized at levels 2-3 log units above the absolute sensitivity level of control fish. The threshold elevation shown by DA-IPC-depleted animals was caused primarily by a loss of rod system function. In DA-IPC-depleted animals, the electroretinograms (ERGs) were normal in terms of absolute light sensitivity and response amplitudes, but the light levels required to elicit ganglion cell discharges were elevated. No evidence of a circadian regulation of behavioral or ERG sensitivity was observed when DA-IPCs were depleted, in contrast to controls. The behavioral visual sensitivity loss of DA-IPC-depleted animals was partially rescued when dopamine or a long-acting dopamine agonist were injected intraocularly. Our data suggest that dopamine is required for maintenance of rod system function as well as for the circadian control of visual sensitivity.

\section{MATERIALS AND METHODS}

Animals and maintenance. Zebrafish were maintained as described (Westerfield, 1995). Zebrafish used in this study were 8-14 months old. Animals were maintained in a 14-10 hr light/dark cycle (light, 8 A.M.-10 P.M.; intensity, $1.15-2.45 \mu \mathrm{W} / \mathrm{cm}^{2}$ ). For some of the circadian experiments, animals were phase-shifted to a new light/dark cycle (light, 10 P.M.-noon), and visual thresholds were evaluated after the animals had been in the shifted light/dark cycle for at least 2 weeks. In the continuous darkness experiments shown in Figures 4-7, visual threshold measurements were made during the first day of continuous darkness.

6-Hydroxydopamine treatment. Methods used for DA-IPC depletion were similar to those as described (Negishi et al., 1982a,b; Lin and Yazulla, 1994a,b). In brief, $2 \mu \mathrm{l}$ of a mixture of 1:1 6-hydroxydopamine 
(6-OHDA) and pargyline (Sigma, St. Louis, MO) (5 $\mu \mathrm{g} / \mu \mathrm{l}$ in PBS) was injected into the vitreous of each eye. The injection was repeated the next day. Visual threshold measurements were made 2 weeks after the injections, except for the recovery experiments shown in Figure 2.

Intraocular injection of dopamine and 2-amino-6, 7-dihydroxy-1, 2, 3, 4-tetrahydronaphthalene hydrobromide. Dopamine and a long-acting dopamine receptor agonist, 2-amino-6, 7-dihydroxy-1, 2, 3, 4-tetrahydronaphthalene hydrobromide (ADTN) (RBI, Natick, MA), were dissolved in PBS before the experiment. One microliter of PBS (control), dopamine $(10 \mu \mathrm{M}, 200 \mu \mathrm{M}$, or $20 \mathrm{mM})$, or $\operatorname{ADTN}(1 \mu \mathrm{M}, 10 \mu \mathrm{M}$, or $1 \mathrm{mM})$ was injected into the vitreous of each eye of 6-OHDA-treated animals (2 week after injection). The fish were kept under normal room illumination and were dark-adapted for $20 \mathrm{~min}$ before threshold measurements.

The behavioral assay. The apparatus used for behavioral analysis of zebrafish visual sensitivity has been described (Li and Dowling, 1997). In brief, zebrafish are placed in a clear container surrounded by a rotating circular drum. A black segment marked on the drum serves as a threatening object. The drum is illuminated from above with a white light (log $0=4.60 \times 10^{2} \mu \mathrm{W} / \mathrm{cm}^{2}$ ) and turned at $10 \mathrm{rpm}$ by a motor. Zebrafish were tested for escape responses when they visually encountered the rotating black segment.

Unless specified otherwise, visual threshold measurements were made during the late afternoon hours (between 4 and 8 P.M.) when the control zebrafish are most sensitive to light (Li and Dowling, 1998). To measure dark adaptation, animals were first light-adapted for $15-20 \mathrm{~min}(3.25 \times$ $\left.10^{3} \mu \mathrm{W} / \mathrm{cm}^{2}\right)$. During subsequent dark adaptation, the minimum light intensity falling on the drum that was required to evoke an escape response when the fish was challenged by the threatening object was recorded. The first threshold measurement was made at 2 min after the start of dark adaptation, then repeated at 2 min intervals, and completed at $26 \mathrm{~min}$ of dark adaptation. Log threshold intensity was plotted as a function of time in the dark. To measure the absolute visual sensitivity, animals were kept in complete dark for a minimum of $20 \mathrm{~min}$ before a threshold measurement was made. To measure incremental sensitivity, fully dark-adapted animals were exposed to a background light for $2 \mathrm{~min}$ (maximum intensity, $4.60 \times 10^{2} \mu \mathrm{W} / \mathrm{cm}^{2}$ ) before a threshold measurement was made. Background illumination was started at the dimmest level, $\log I=-8.0$, and was increased by steps of $1 \log$ unit. The threshold measurement was made within $10 \mathrm{sec}$ after the background light was turned off.

Immunocytochemistry. Methods used for immunocytochemistry were similar as described (Schmitt and Dowling, 1996). Specimens were fixed in $4 \%$ paraformaldehyde in PBS overnight. After they were blocked with $10 \%$ normal goat serum, specimens were incubated with primary antiserum [tyrosine hydroxylase (TH), 1:200 (Chemicon, CA); 5E11, 1:10, provided by J. Fadool] overnight and were then incubated with secondary antibody (FITC-conjugated, 1:50; Boehringer Mannheim, Indianapolis, IN) for $2 \mathrm{hr}$. Specimens were mounted on slides and viewed by fluorescence microscopy.

ERG and ganglion cell recordings. Methods used for ERG recordings have been described (Li and Dowling, 1997, 1998). Zebrafish were anesthetized with 4\% 3-amino benzoic acid methylester and immobilized with $10 \%$ gallamine triethiodide. Zebrafish were dark-adapted for 20-30 min before an ERG was recorded. The fish were illuminated with 500 msec flashes (full field, maximum intensity, $6.75 \times 10^{3} \mu \mathrm{W} / \mathrm{cm}^{2}$ ). The electrical signals were recorded using a glass pipette (filled with a balanced salt solution) placed on the center of the cornea.

Ganglion cell action potentials were recorded from the optic nerve using a tungsten microelectrode (Frederick Haer, Inc.). Fish were anesthetized and immobilized as described above. The connective tissue surrounding the eye was cut using a pair of microsurgery scissors. The eye was pulled out of the orbit slightly (by 1-2 mm) and held with two glass rods to expose the optic nerve. The fish were illuminated with 500 msec white light flashes (full field, the same light source used for ERG recordings). The ganglion cell discharge was amplified and recorded conventionally. In most cases, we recorded ganglion cell discharges simultaneously from four to six cells. Our criterion for threshold was the observation of an obvious increase in ganglion cell discharge on the oscilloscope.

\section{RESULTS}

\section{Depletion and regeneration of retinal DA-IPCs}

Retinal DA-IPCs were depleted by intraocular injections of 6-OHDA. The depletion and subsequent regeneration of DA-
IPCs were monitored by examining the cells immunoreactive to TH in the retina (Negishi et al., 1982a,b; Lin and Yazulla, 1994a,b). To ensure that other retinal neurons were unaffected by 6-OHDA injections, we examined 6-OHDA-treated retinas using conventional histological and immunocytochemical methods [with 5E11, an antibody against zebrafish amacrine cells (Fadool et al., 1999)]. No obvious differences in the number and appearance of other inner retinal neurons were observed between control and 6-OHDA-treated retinas.

Figure 1 shows confocal images of DA-IPCs taken from the nasal/ventral region of flat-mounted retinas. In control animals, DA-IPCs were found scattered quite evenly across much of the retina. The total number of DA-IPCs in control retinas varied somewhat from animal to animal, from $\sim 1100$ to 1300 cells per retina $(n=12)$. At 2 weeks after injection, virtually all of the DA-IPCs were gone, although an occasional TH-positive cell was observed (Fig. 1C). DA-IPCs gradually regenerated after the 6-OHDA treatment. At 8 weeks after injection, for example, regenerated DA-IPCs were seen in the peripheral regions of the retina (Fig. 1D). The regeneration of DA-IPCs continued over time. By 24 weeks after injection, DA-IPCs were observed in both the peripheral and middle regions of the retina. However, the regeneration of DA-IPC was not complete even after 40 weeks of 6-OHDA treatment. At 9 months after injection, for example, only $\sim 650$ DA-IPCs were observed (Fig. $1 E)(n=2)$. Furthermore, most of the regenerated DA-IPCs $(>450)$ were found in the peripheral outer third of the retina. TH-positive processes that grew out from regenerated DA-IPCs were observed projecting into the central regions of the retina.

The somata of newly regenerated DA-IPCs appeared to be smaller than those of DA-IPCs in control fish. The somata of DA-IPCs observed in the peripheral regions of 2 and 8 week post-injected retinas were $\sim 7-11 \mu \mathrm{m}$ in diameter (Fig. $1 C, D$ ), whereas they were $\sim 13-17 \mu \mathrm{m}$ in the same region of the control fish (Fig. 1B). Over time, the somata of regenerated DA-IPCs became larger and eventually were comparable in size to those of control fish (Fig. 1E). At 9 months after injection, TH-positive processes that grew out from regenerated DA-IPCs (Fig. 1G) were similar in appearance to those of the control animals (Fig. $1 F)$.

\section{Rod system function is not evident in dark-adapted DA-IPC-depleted animals}

We evaluated behaviorally the visual sensitivity of 6-OHDAtreated animals. During dark adaptation after bright light adaptation, we measured the light thresholds needed to evoke escape responses when the fish were challenged by a threatening object. The first threshold measurement was made 2 min after the start of dark adaptation, then repeated in 2 min intervals, and completed at 26 min of dark adaptation (Li and Dowling, 1997).

The results are shown in Figure $2 A$. Thresholds determined between 2 and 8 min of dark adaptation were not significantly different between control and 6-OHDA-treated animals (2, 8, and 16 weeks after injections; $n=12$ for each group). However, between 8 and 14 min of dark adaptation, visual thresholds of 2 week post-injection animals $(\square)$ increased somewhat. In contrast, the behavioral visual thresholds of control fish (0) decreased substantially until they plateaued at 20 min of dark adaptation, at which time they were $\sim 3 \log$ units below the final threshold levels of 2 week post-injection fish (Fig. 2A). With regeneration of DA-IPCs in the retina, visual thresholds of 6-OHDA-treated fish gradually recovered. By 8 weeks after injection $(\triangle)$, the final 

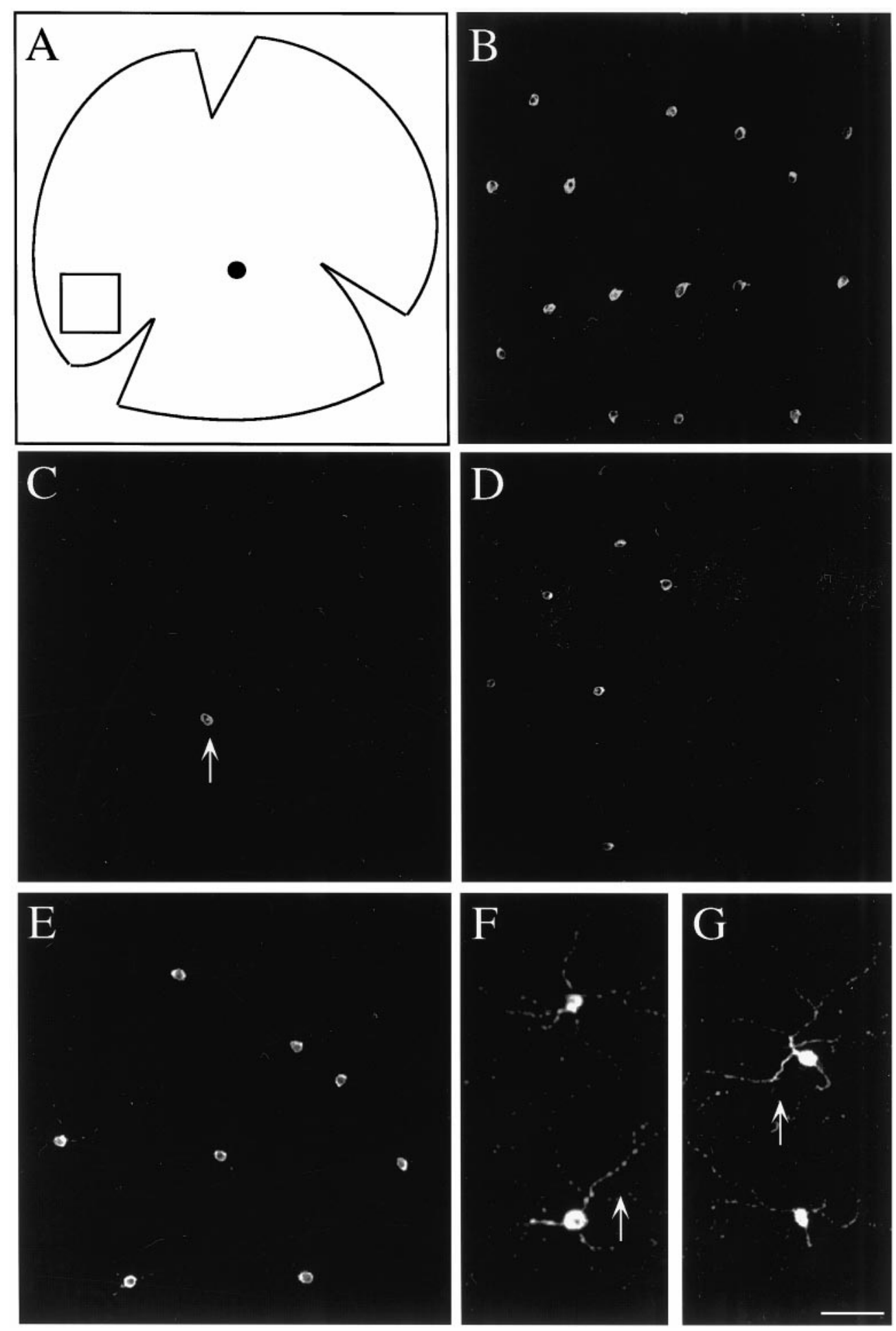

Figure 1. Confocal images of DAIPCs. $A$, A schematic drawing of a flatmounted retina. Dorsal is up, and anterior is to the left. The filled circle roughly in the center of the retina indicates the optic disk. The box drawn in the nasal/ ventral region of the retina indicates the location of confocal images shown in $B-G$. $B$, A confocal image showing DAIPCs from a control retina. DA-IPCs (bright staining) were stained with an antibody against tyrosine hydroxylase. $C-E$, Confocal images showing the retina at 2 weeks $(C), 16$ weeks $(D)$, and 9 months $(E)$ after the 6-OHDA treatment. At 2 weeks after injection, only an occasional TH-positive cell was detected in the retina (arrow). At 16 weeks and 9 months, more TH-positive cells were observed, especially in the peripheral regions of the retina. $F, G$, Enlarged pictures showing DA-IPCs as well as their processes in control $(F)$ and 9 month post-injection 6-OHDA treated $(G)$ retinas. Scale bar: $B-E, 80 \mu \mathrm{m} ; F, G, 40 \mu \mathrm{m}$.

thresholds were $\sim 2 \log$ units above control thresholds, and by 16 weeks $(\bigcirc)$, thresholds determined at $20 \mathrm{~min}$ of dark adaptation were similar to those of control fish (O) (Fig. 2A). Visual thresholds of four sham-injected zebrafish (injected with PBS) were also evaluated using the same procedure as described above. No obvious differences in behavioral visual sensitivity were observed between sham-injected and control fish (data not shown).

The final visual thresholds of 2-week post-injection fish (referred to as DA-IPC-depleted) were raised by 2-3 log units and reached levels comparable to cone system thresholds in control fish (Fig. 2A). To determine whether the threshold elevation in DA-IPC-depleted animals is caused by a loss of rod system function, we measured the course of dark adaptation using red $(625 \mathrm{~nm})$ and green $(500 \mathrm{~nm})$ illumination that was closely matched phototopically. The results are shown in Figure $2 B$. In control animals $(n=8)$, thresholds determined in green light $(\bigcirc)$ were slightly lower during the first 6 min of dark adaptation as compared with the thresholds determined in red light $(\bigcirc)$, but thereafter they deviated substantially. For example, the threshold differences determined with the red and 

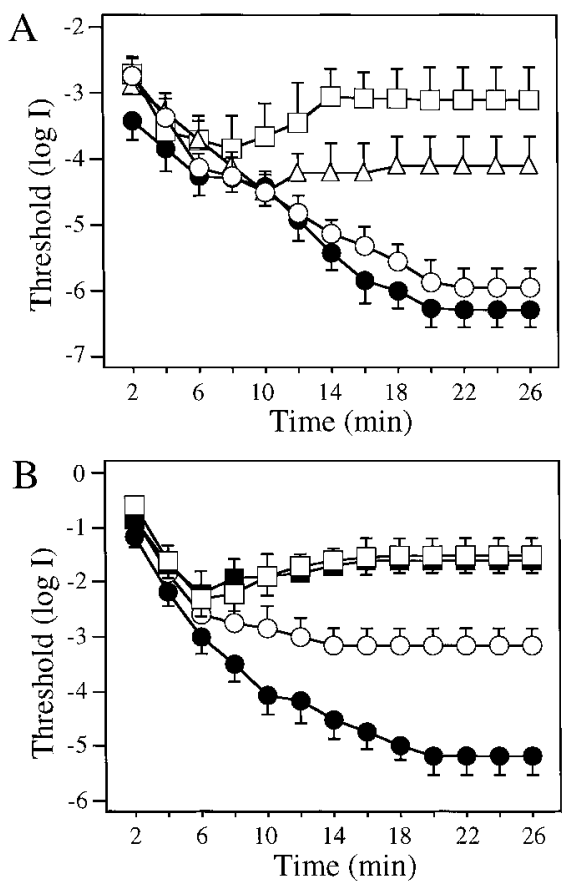

Figure 2. Dark adaptation curves determined using white $(A)$ and colored $(B)$ illumination of the rotating drum. $A$, Dark adaptation curves of control $(\bullet, n=12)$ and 6-OHDA-treated animals $(\square, 2$ weeks after injection; $\triangle, 8$ weeks after injection; $\bigcirc, 16$ weeks after injection; $n=12$ for each group). Between 2 and 8 min of dark adaptation, thresholds measured in 6-OHDA-treated animals were similar to thresholds measured in control fish. Note the rise in thresholds in 2 and 8 week post-injection animals between 8 and 14 min of dark adaptation. $B$, Dark adaptation curves determined using red $(625 \mathrm{~nm}$, open symbols $)$ and green $(500 \mathrm{~nm}$, filled symbols) illumination of the rotating drum in control (circles, $n=8$ ) and 6-OHDA-treated ( 2 weeks after injection; squares, $n=$ 8) animals. In control animals, the threshold difference determined using these two filters was $<0.3 \log$ units at 6 min but $>2 \log$ units at 20 min of dark adaptation. At all times during dark adaptation, no threshold differences with red or green illumination were observed in 6-OHDA-treated animals. Note the threshold elevation in 6-OHDA-treated fish between 6 and 16 min of dark adaptation. Data represent the means \pm SE.

green lights were $<0.3 \log$ units when measured at 6 min of dark adaptation, but they increased to $>2.0 \mathrm{log}$ units when measured at 20 min of dark adaptation. This is the classic Purkinje phenomenon; during early dark adaptation, retina is relatively red sensitive (governed by cones) and later it is more green sensitive (governed by rods) [see also Li and Dowling (1998)].

At all times during dark adaptation, visual thresholds determined with the red $(\square)$ and green $(\square)$ lights were similar in DA-IPC-depleted animals $(n=8)$ (Fig. $2 B$ ). For the first $6 \mathrm{~min}$ in the dark, visual thresholds of DA-IPC-depleted fish decreased; thereafter, they increased until they plateaued at 14-16 min of dark adaptation. The finding that DA-IPC-depleted animals were equally sensitive to the red and green lights during the entire course of dark adaptation suggests that the rod system is not functioning in the absence of dopamine in the retina. Furthermore, the fact that the final visual thresholds (measured at $20 \mathrm{~min}$ of dark adaptation) of DA-IPC-depleted animals were higher than the thresholds measured at $6-8$ min of dark adaptation suggests that there is also a cone system deficit in dark-adapted animals.
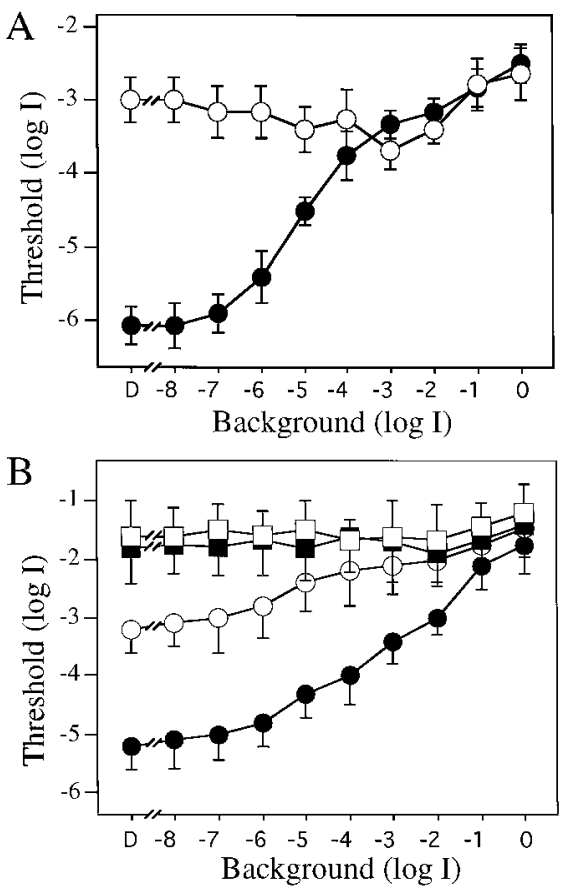

Figure 3. Incremental sensitivity curves determined using white $(A)$ and colored $(B)$ illumination of the rotating drum. $A$, Visual thresholds of control $(\bullet, n=6)$ and DA-IPC-depleted $(\bigcirc, n=6)$ animals measured behaviorally with white light illumination of the drum. With dim background illumination (between $\log I=-8.0$ and -7.0 ), the visual thresholds of control animals were $\sim 3 \log$ units lower than the visual thresholds of DA-IPC-depleted animals. After bright background illumination (between $\log I=-4.0$ and $\log I=0)$, the thresholds were similar between these two groups. $B$, Incremental sensitivity of control (circles, $n=6)$ and DA-IPC-depleted animals (squares, $n=6)$ using green $(500 \mathrm{~nm}$, filled symbols) or red (625 nm, open symbols) illumination of the rotating drum. In control animals, the threshold difference determined using these two filters was $\sim 2 \log$ units under dim background light $(\log I<-5.0)$ but was less than a half $\log$ unit under bright background light $(\log I>-1.0)$. In DA-IPC-depleted animals, no threshold differences were observed using green and red illumination. Data represent the means \pm SE.

\section{Cone system function is normal in DA-IPC-depleted animals in bright light}

We next evaluated incremental sensitivity of control and DAIPC-depleted fish. Animals were kept in complete darkness for $20 \mathrm{~min}$ before the incremental sensitivity measurements were made. Background illumination was started at $\log I=-8.0$ (maximum light, $4.60 \times 10^{2} \mu \mathrm{W} / \mathrm{cm}^{2}$ ) and increased by steps of $1 \log$ unit.

The results are shown in Figure $3 A$. With very dim background illumination, i.e., $\log I=-8.0$, the absolute thresholds measured in control $(\Theta, n=6)$ and DA-IPC-depleted animals $(\bigcirc, n=6)$ were similar to those measured in complete darkness; the thresholds of DA-IPC-depleted animals were $\sim 3 \log$ units higher than the thresholds of control animals. With increases in background illumination, the thresholds measured in control animals rose gradually. With background illumination of $\log I=-5.0$, for example, the thresholds of control fish were elevated $\sim 1.5 \log$ units as compared with the thresholds measured in complete darkness.

The thresholds of DA-IPC-depleted animals, on the other hand, remained virtually unchanged as the background illumination increased (Fig. 3A). Visual thresholds of control and DAIPC-depleted animals thus gradually converged as the back- 
Table 1. Thresholds of ERG and retinal ganglion cell spikes of control and DA-IPC-depleted animals $(\log \mathrm{I})$

\begin{tabular}{lll} 
& ERG & Ganglion cells \\
\hline Control & $-6.1 \pm 0.2$ & $-6.4 \pm 0.2$ \\
DA-IPC-depleted & $-5.9 \pm 0.2$ & $-4.6 \pm 0.3$
\end{tabular}

For ERG recordings, $n=6$ for both the control and DA-IPC-depleted animals. For ganglion cell recordings, $n=12$ for the control fish and $n=10$ for DA-IPC-depleted fish. Data represent the means \pm SE.

ground illumination was increased, as a result of a substantial threshold increase in the control fish along with a slight threshold decrease in DA-IPC-depleted fish. With bright background illumination $(\log I>-3.0)$, the thresholds of control and DA-IPCdepleted animals were similar; they then rose together as the background illumination was increased further (Fig. $3 A$ ).

We next measured incremental sensitivity of control and DAIPC-depleted animals using phototopically matched red and green lights. In controls, after exposure to dim levels of white light background illumination (between $\log I=-8.0$ and $\log I=$ $-6.0)$, the thresholds determined with the green light $(500 \mathrm{~nm}, 0)$ were $\sim 2 \log$ units lower than the thresholds determined with the red light $(625 \mathrm{~nm}, \bigcirc)$ (Fig. $3 B)$. These results indicate that retinal function is governed by rods under dim background illumination. As the background illumination was increased, the threshold differences determined using the green and red lights became smaller. With the brightest background illumination $(\log I=-1.0$ and 0 ), thresholds determined in control animals with the red and green lights were similar, indicating that visual sensitivity was now controlled by the cone system. In DA-IPC-depleted fish, thresholds determined in bright $(\log I=-1.0$ and 0$)$ red $(\square)$ and green $(\square)$ light were virtually identical to those of the control fish, suggesting that the cone system function was normal in bright light. Rod system function, however, was never evident in DAIPC-depleted animals; at dimmer levels of background illumination $(\log I<-3.0)$, thresholds measured with the red and green lights were always similar in DA-IPC-depleted fish (Fig. $3 B$ ).

\section{Outer retinal function in DA-IPC-depleted animals appears normal}

We next measured ERG sensitivity to determine whether the loss of rod system function in DA-IPC-depleted animals is caused by a dysfunction in the outer retina. In these experiments, we measured the threshold light intensity that was required to elicit a threshold ERG (10-20 $\mu \mathrm{V}$ b-wave) using fully dark-adapted animals. Animals were kept in complete darkness for $\sim 30 \mathrm{~min}$ before a threshold ERG was recorded. The ERG responses were averaged four to six times to increase the signal-to-noise ratio. The light intensity that was required to elicit threshold ERGs was similar between control $(n=6)$ and DA-IPC-depleted animals $(n=6)$ (Table 1$)$, suggesting that outer retinal function was normal in the absence of dopamine in the retina.

The ERGs also appeared normal in DA-IPC-depleted animals when measured with red and green lights. They showed normal threshold levels as well as the Purkinje phenomenon. At 6 min of dark adaptation, for example, the ERG threshold differences determined with red and green lights were $\sim 0.3$ log units, similar to those of controls (Table 2). At 20 min of dark adaptation, the threshold differences as determined by red and green lights had increased to $1.2 \mathrm{log}$ units, again, similar to those of control animals (Table 2).
Table 2. Threshold differences (log I) of ERG sensitivity determined by the red and green lights during dark adaptation

\begin{tabular}{lll} 
& $6 \mathrm{~min}$ & $20 \mathrm{~min}$ \\
\hline Control & 0.1 & 1.4 \\
DA-IPC-depleted & 0.3 & 1.2
\end{tabular}

Experiments were performed in the late afternoon hours. $n=6$ for both the control and DA-IPC-depleted animals.

\section{Control}

6-OHDA
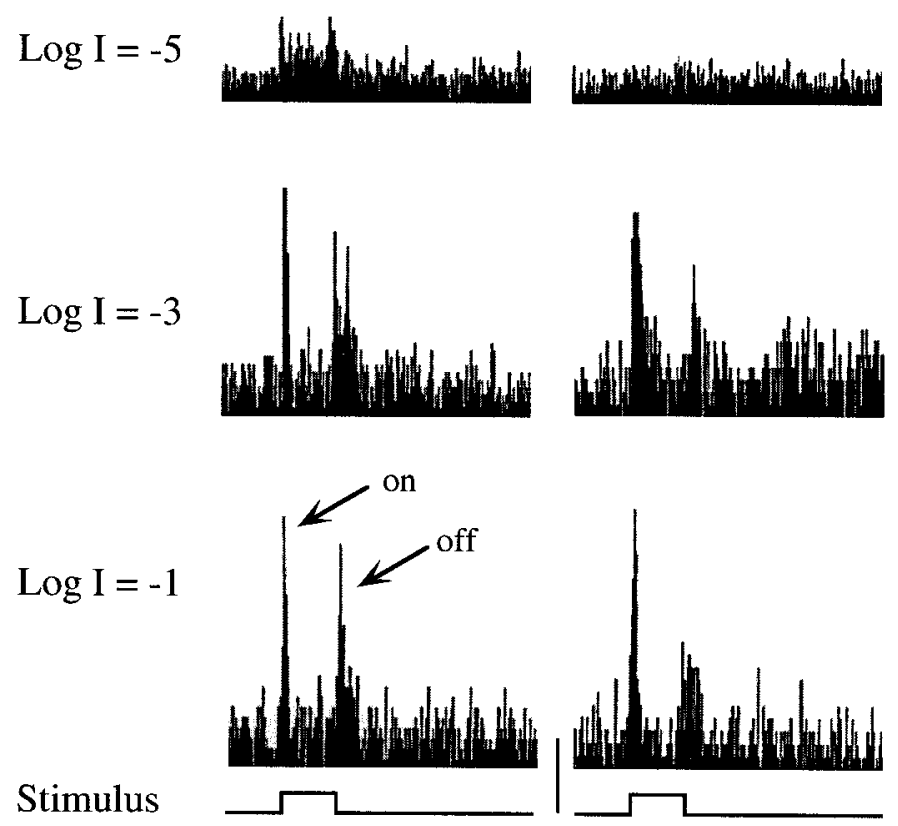

Figure 4. Representative ganglion cell discharge histograms from control (left) and DA-IPC-depleted zebrafish (right). Light responses to 0.5 sec of bright $(\log I=-1.0)$, medium $(\log I=-3.0)$, and $\operatorname{dim}(\log I=$ $-5.0)$ illumination were recorded from the optic nerve. With bright and medium illumination, ganglion cell $\mathrm{ON}$ and $\mathrm{OFF}$ responses were recorded from both control and DA-IPC-depleted fish. No obvious ganglion cell discharge was observed in DA-IPC-depleted animals when tested with dim illumination, unlike the control fish in which both ON and OFF responses were recorded, along with some sustained discharge, to this light level. on, ON responses; off, OFF responses. Calibration (bottom center): 40 spikes/sec.

\section{Inner retina function of DA-IPC-depleted animals is abnormal}

To determine whether the threshold elevation in DA-IPCdepleted animals is caused by abnormalities occurring in the inner retina, we measured threshold light intensities that were required to elicit a retinal ganglion cell discharge in the optic nerve (Fig. 4). In most cases, we recorded simultaneously from four to six cells. Our criterion for ganglion cell threshold was the observation of an increase of ganglion cell discharge on the oscilloscope screen.

Threshold light intensities that were required to fire action potentials in retinal ganglion cells were elevated in DA-IPCdepleted animals. On average, the threshold was $1.8 \log$ units higher in DA-IPC-depleted animals $(n=10)$ than in control animals $(n=12)$ (Table 1$)$. This result suggests that the visual defect caused by DA-IPC depletion occurs primarily in the inner retina. 


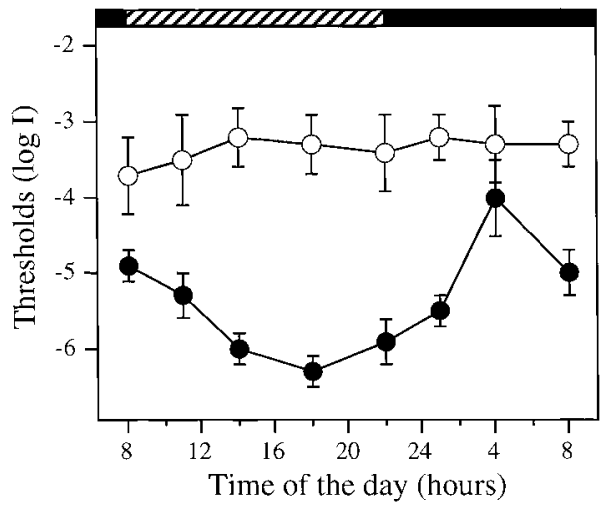

Figure 5. Absolute visual sensitivity of control $(\bullet, n=6$ for each time point) and DA-IPC-depleted fish $(O, n=6$ for each time point) measured behaviorally as function of time of day. Animals were kept in DD during the experiment. Note the threshold variation in the control fish between subjective day and night. In DA-IPC-depleted fish, on the other hand, the behavioral visual sensitivity was maintained at a constant level, $\sim 3.0 \mathrm{log}$ units above the most sensitive level of the control fish. Horizontal black bars indicate subjective night; hatched bars indicate subjective day. Data represent the means $\pm \mathrm{SE}$.

\section{The circadian rhythm of behavioral and ERG sensitivity is lost in DA-IPC-depleted animals}

To determine whether the circadian rhythm of visual sensitivity persists in the absence of dopamine in the retina, we evaluated visual sensitivity of DA-IPC-depleted animals as a function of time of day while animals were kept in constant darkness (DD). We measured visual thresholds behaviorally as well as by the ERG. For behavioral threshold measurements, the experiments were repeated at 3-4 hr intervals over a $24 \mathrm{hr}$ period. In controls $(\bullet, n=6)$, the behavioral visual thresholds fluctuated systematically between subjective day and night; they were highest at subjective dawn and lowest at subjective dusk (Fig. 5). In DAIPC-depleted animals $(\bigcirc, n=6)$, on the other hand, visual thresholds were maintained at a constant level; at all times during subjective day and night, they were 2-3 log units higher than the thresholds measured at subjective dusk in control animals (Fig. 5).

ERG thresholds were measured at four time points: subjective dawn, noon, dusk, and midnight, over a $24 \mathrm{hr}$ period. We recorded ERG sensitivity of both the rod and cone systems. For rod sensitivity measurements, animals were kept in complete darkness. The only light the fish were exposed to was the test light, which was kept close to threshold levels. For cone sensitivity measurement, dark-adapted animals were exposed to background light for 2-3 min before a threshold measurement was made (log $I=-3.0$, the same background light used for incremental sensitivity measurement; under this level of illumination, no behavioral rod system function was evident) (Fig. 3A). The threshold light that was required to elicit a threshold ERG $(10-15 \mu \mathrm{V}$ b-wave) was recorded immediately (within $10 \mathrm{sec}$ ) after the background light was turned off.

The results are shown in Figure $6 A, B$. In control animals (-) $n=6$ for each time point), both the rod and cone thresholds fluctuated by $\sim 1.0 \log$ units between subjective dawn and dusk. They were highest at dawn, then fell gradually, reaching their lowest levels at dusk, and were higher again at subjective midnight. Circadian regulation of the rod and cone system ERG sensitivity was abolished in DA-IPC-depleted animals. In DAIPC-depleted animals $(\bigcirc, n=6$ for each time point), both the rod and cone thresholds were maintained at a constant level through-
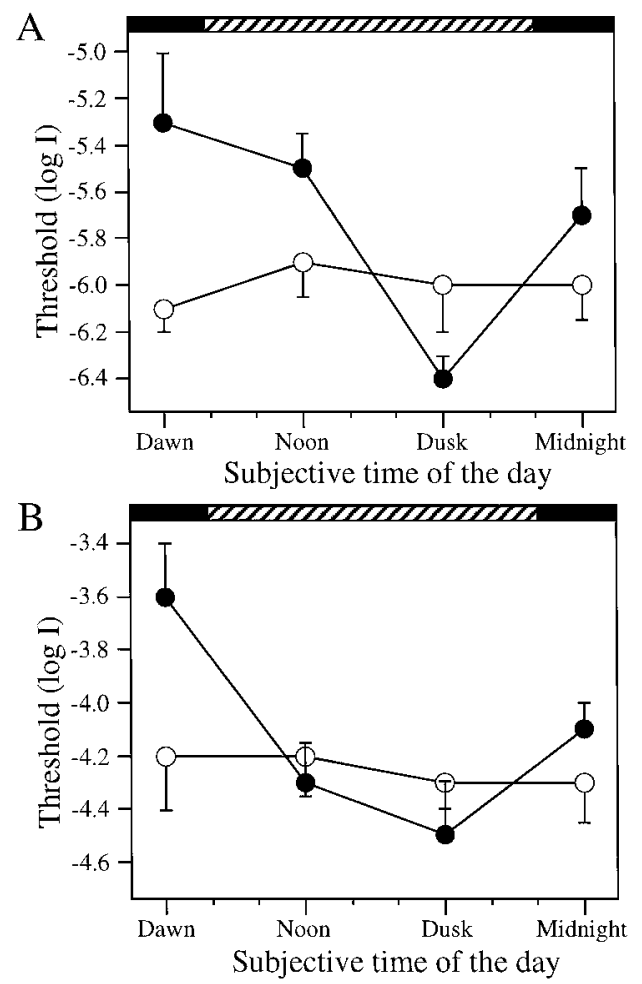

Figure 6. Rod $(A)$ and cone $(B)$ sensitivity determined by the ERG as function of time of day. Animals were kept in DD during the experiment. $A$, ERG sensitivity of rods. Note the systematic threshold variation in control fish $(\bullet, n=6$ for each time point) between subjective day and night. No threshold variation was seen in DA-IPC-depleted retinas $(\bigcirc$, $n=6$ for each time point). $B$, ERG sensitivity of cones. Animals were light-adapted with $\log I=-3.0$ for 2 min before the test. Threshold variation as a function of time of day was observed in the control fish $(\bullet, n=6$ for each time point) but was not observed in DA-IPCdepleted fish $(\bigcirc, n=6$ for each time point). Horizontal black bars indicate subjective night; hatched bars indicate subjective day. Data represent the means \pm SE.

out the day and night, close to the threshold levels measured at subjective dusk in control animals.

The ERG waveforms were similar between control and DAIPC-depleted animals. Figure 7 shows representative ERGs of a control and a DA-IPC-depleted fish recorded at subjective dawn and at dusk. At subjective dusk, both ON and OFF responses were observed. At subjective dawn, however, only $\mathrm{ON}$ responses were recorded. We plotted the ERG b-wave amplitude over $4 \log$ units of light intensity, a $V$-log $I$ curve. In control animals ( $n=6$ at each time for each group), ERG b-wave amplitudes generated at all light intensities were reduced at subjective dawn ( $\square$ ) as compared with those generated at subjective dusk (- Fig. $8 A)$. The $V$-log $I$ curve determined at subjective dawn was shifted to the right on the intensity axis by $1.0 \log$ units as compared with the $V$ - $\log I$ curve determined at subjective dusk. ERG $V$-log $I$ curves of DA-IPCdepleted animals, on the other hand, were similar when determined at subjective dawn $(\square)$ and dusk $(\bigcirc, n=6$ at each time for each group) (Fig. 8B). The ERG thresholds of DA-IPC-depleted animals were similar to those of control animals determined at subjective dusk, but the maximal ERG amplitudes were reduced in DA-IPC-depleted animals as compared with those of control animals. 


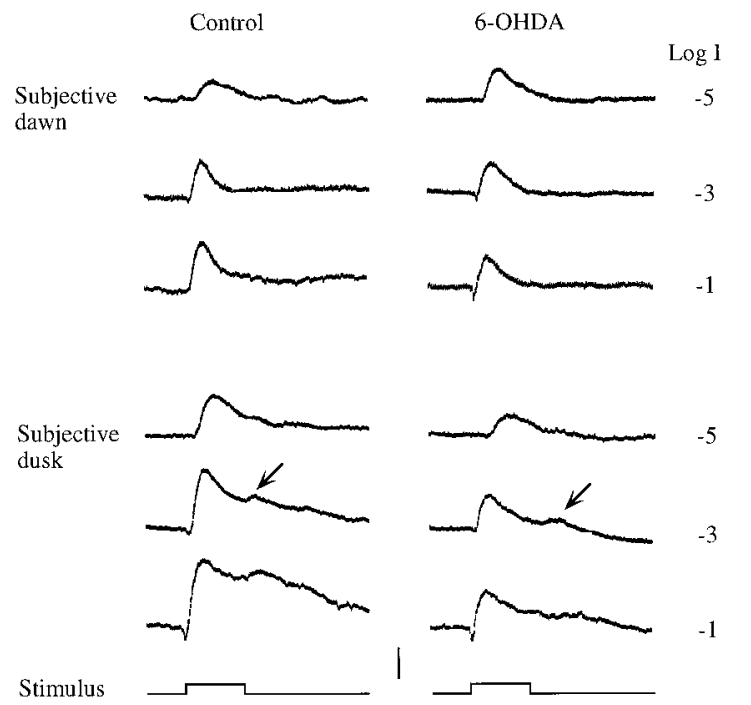

Figure 7. Representative ERGs recorded from a control and a DA-IPCdepleted fish at subjective dawn and dusk in animals kept in DD. Note at subjective dusk, both ON and OFF responses were recorded in both the control and DA-IPC-depleted fish. No OFF responses were evident at subjective dawn in either fish. OFF responses are indicated by arrows. Stimuli, $500 \mathrm{msec}$. Calibration, $100 \mu \mathrm{V}$.

\section{Dopamine and a dopamine agonist rescue partially the loss of visual sensitivity of DA-IPC-depleted animals}

Both behavioral and electrophysiological findings presented in this paper suggest that dopamine is required for rod signal transmission through the inner plexiform layer of the retina. An obvious question is whether dopamine exogenously applied to the retina can reverse the loss of visual sensitivity observed in DAIPC-depleted animals. To this end, intraocular injections of dopamine and a long-acting dopamine agonist, ADTN (Miller et al., 1974; Nicola et al., 1996), were performed. One microliter of PBS (for control), dopamine, or ADTN was injected into each eye of the DA-IPC-depleted animals. Behavioral threshold measurements were made at $0.5,1$, and $2 \mathrm{hr}$ (for PBS and dopamine injections) and 1,2 , and $4 \mathrm{hr}$ after injections (for ADTN injections).

Both dopamine and ADTN significantly decreased the behavioral visual threshold of DA-IPC-depleted animals when applied at moderate concentrations. Thirty minutes after the injection of dopamine $(200 \mu \mathrm{M}), 6$ of 10 animals showed visual threshold decreases equal to or greater than $0.5 \mathrm{log}$ units as compared with light threshold measured before dopamine injections. At $30 \mathrm{~min}$ and $1 \mathrm{hr}$ after the dopamine injection, the average visual threshold of DA-IPC-depleted animals was 0.5 and $0.3 \log$ units, respectively, below the threshold level measured before dopamine injections. An effect of dopamine on visual sensitivity was not obvious at $2 \mathrm{hr}$ after injection (Fig. 9B).

Intraocular injections of ADTN decreased the visual thresholds of DA-IPC-depleted animals more convincingly. At $1 \mathrm{hr}$ after $\operatorname{ADTN}(10 \mu \mathrm{M})$ injections, 12 of 16 tested fish showed threshold decreases equal to or greater than $0.5 \log$ units as compared with thresholds measured before ADTN injections. The average visual threshold measured at 1 and $2 \mathrm{hr}$ after injection was $1.0 \mathrm{log}$ unit below the threshold level measured before ADTN injections. The effects of ADTN on behavioral visual sensitivity decreased with time. At $4 \mathrm{hr}$ after injection, the behavioral visual thresholds of DA-IPC-depleted animals had
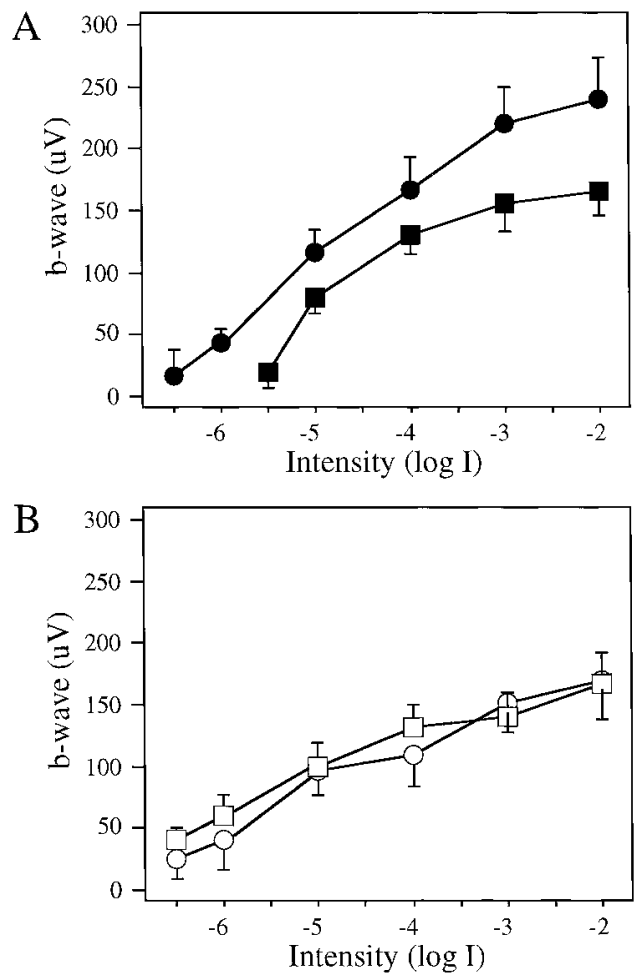

Figure 8. $\quad V-\log I$ curves of control $(A)$ and DA-IPC-depleted $(B)$ fish at subjective dawn and dusk ( $n=6$ for each time point). Animals were kept in DD during the experiment. $A$, In control fish, the ERG sensitivity was reduced by $\sim 1 \log$ unit at subjective dawn $(\boldsymbol{\square})$ as compared with ERG sensitivity at subjective dusk (); the $V$-log $I$ curve determined at dawn was shifted to the right by $\sim 1 \log$ unit as compared with that determined at dusk. $B$, No ERG sensitivity changes were observed in DA-IPCdepleted animals between subjective dawn $(\square)$ and dusk $(\bigcirc)$. Note that the $V$ - $\log I$ curves were similar between dawn and dusk in DA-IPCdepleted fish. Data represent the means \pm SE.

returned to levels similar to those measured before ADTN injections (Fig. 9C).

Intraocular injections of either a low or high concentration of dopamine or ADTN produced no or little effect on behavioral visual thresholds of DA-IPC-depleted animals. For example, 30 min after an injection of dopamine $(10 \mu \mathrm{M}$ or $20 \mathrm{mM})$, the behavioral visual thresholds of DA-IPC-depleted animals $(n=$ 10) remained virtually unchanged. ADTN applied at $1 \mu \mathrm{M}$ caused no effect on behavioral visual sensitivity, whereas ADTN at $1 \mathrm{~mm}$ concentration decreased only slightly the visual thresholds of DA-IPC-depleted animals. At $1 \mathrm{hr}$ after ADTN injection (1 mM), the average visual threshold of DA-IPC-depleted animals $(n=6)$ was $0.2 \log$ units below the threshold level measured before ADTN injections. Intraocular injections of PBS (sham injection, $n=10$ ) produced no effect on behavioral visual sensitivity of DA-IPC-depleted animals (Fig. 9A).

\section{DISCUSSION}

We have measured visual sensitivity behaviorally as well as by the ERG and ganglion cell discharge in zebrafish in which the retinal DA-IPCs were depleted by treatment with 6-OHDA. During the course of dark adaptation after bright light adaptation, the behavioral visual thresholds of DA-IPC-depleted animals were similar to those of control fish only for the first 6-8 min; thereafter, they increased and stabilized 2-3 log units above the absolute sensitivity levels of control fish. In DA-IPC-depleted ani- 
$\mathbf{A}$

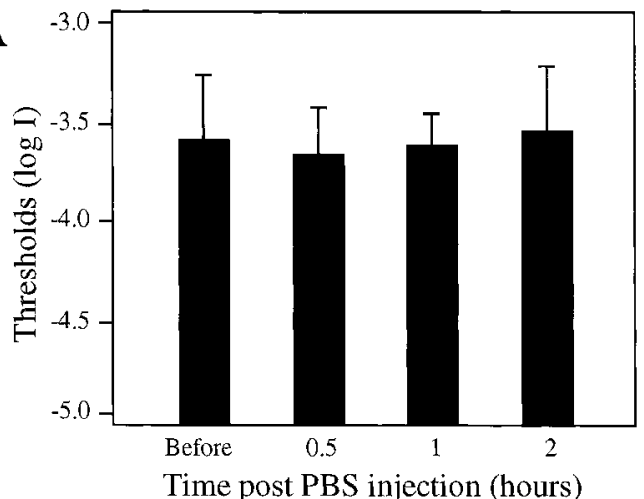

B

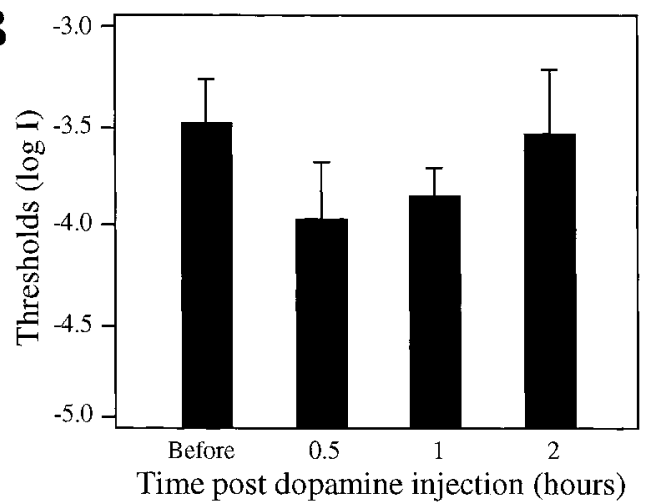

C

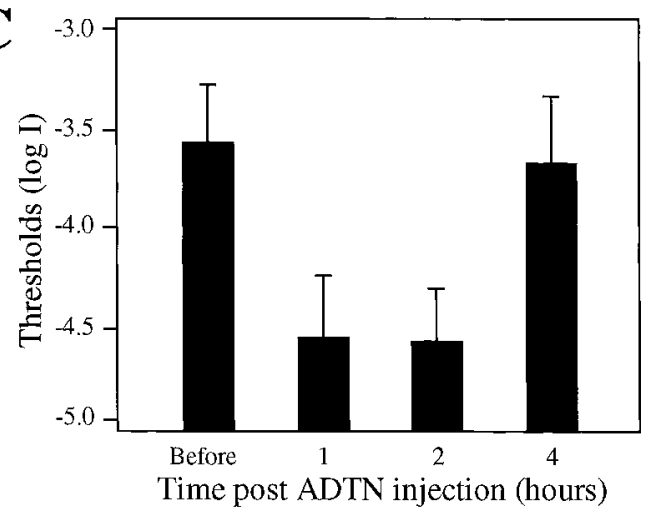

Figure 9. Behaviorally measured visual thresholds of DA-IPC-depleted animals before and after intraocular injections of PBS $(A)$, dopamine $(B)$, and the dopamine receptor agonist $\operatorname{ADTN}(C)$. A, As a control, fish $(n=$ 10) were sham-injected with PBS. No significant visual threshold changes were observed after PBS injections. $B$, Dopamine intraocularly injected at a concentration of $200 \mu \mathrm{M}$. Note the threshold decreases at $30 \mathrm{~min}$ and 1 $\mathrm{hr}$ after dopamine injection $(n=10 ; p<0.001)$. At $2 \mathrm{hr}$ after injection, the thresholds of DA-IPC-depleted animals returned to levels similar to those measured before dopamine injections. $C$, ADTN intraocularly injected at a concentration of $10 \mu \mathrm{M}$. At 1 and $2 \mathrm{hr}$ after injection, visual thresholds of DA-IPC-depleted animals $(n=16)$ were significantly decreased $(p<0.001)$. The effect of ADTN on behavioral visual sensitivity was insignificant at $4 \mathrm{hr}$ after injection. Data represent the means \pm SE.

mals, the threshold elevation was observed under dim background illumination. However, after bright background illumination, visual thresholds of DA-IPC-depleted animals and control animals were similar. By probing the spectral sensitivity using phototopically matched red and green lights, we found that the threshold elevation in DA-IPC-depleted animals was caused primarily by a loss of rod system function. ERG measurements indicate that the rod photoreceptor cells themselves appear to function properly in
DA-IPC-depleted animals. Recordings of the ganglion cell discharge suggest that the loss of rod system function is caused by effects occurring in the inner retina; rod signals appear to be blocked in the inner plexiform layer in DA-IPC-depleted animals.

\section{Is dopamine a retinal light signal?}

Our results were unanticipated and surprising in view of the generally held view that dopamine serves as a light signal in the retina. For example, dopamine suppresses rod input while enhancing and speeding up cone input in amphibian horizontal cells (Witkovsky and Dearry, 1991; Witkovsky et al., 1993), and a number of studies indicate that dopamine is released in the retina in the light (Bauer et al., 1980; Godley and Wurtman, 1988; Witkovsky et al., 1993). Thus, in the absence of dopamine, one would predict that the retina is rod-dominated, just the opposite of our findings. In DA-IPC-depleted zebrafish retinas, we find that rod system function is dramatically suppressed as measured behaviorally.

We are unaware of any other behavioral studies examining rod visual sensitivity in DA-IPC-depleted animals. In humans with Parkinson's disease in which retinal dopamine levels are presumably depressed, a decrease of contrast sensitivity has been reported (Bodis-Wollner et al., 1984, 1987; Bodis-Wollner, 1990; Masson et al., 1993), but no reports on rod sensitivity in such patients have appeared. In studies of ganglion cells in 6-OHDAtreated animals, alterations in receptive field organization have been described, but these experiments were performed in the presence of background light and did not examine rod system sensitivity [Jensen and Daw, 1984, 1986; Maguire and Smith, 1985 (but see Maguire and Hamasaki, 1994)]. Lin and Yazulla (1994a) examined the tilting behavior of goldfish treated with 6-OHDA. They found that under uniform overhead illumination, the goldfish tilted toward the side of the unilaterally injected eye, suggesting that the light sensitivity of the injected eye was increased. However, the tilting behavior was observed only under lightadapted conditions. Within 2 min of dark adaptation, the tilting disappeared, indicating that the tilting phenomenon was cone system-mediated.

One might question whether our behavioral test is truly measuring rod system sensitivity or some other phenomenon. For example, we are asking the animal to distinguish a black segment on a rotating drum from surrounding regions whose brightness is varied. Thus, both contrast and movement are involved in the detection task. In patients suffering from Parkinson's disease, a decrease in contrast sensitivity has been reported (BodisWollner, 1990; Masson et al., 1993), but it is hard to relate these findings to the present results. That we can plot out classic dark adaptation and incremental sensitivity curves with both cone and rod limbs using our behavioral test [see also Li and Dowling (1997, 1998) and Figs. 2, 3] suggests that our technique does measure light sensitivity of the rod and cone systems in zebrafish.

That we can partially rescue the loss of behavioral visual sensitivity of DA-IPC-depleted fish by an ocular injection of dopamine or a dopamine agonist provides evidence that dopamine is indeed required for the maintenance of light sensitivity in zebrafish. Do our other findings match earlier results? Both Lin and Yazulla (1994b) and Maguire and Smith (1985) found that the sensitivity and waveform of ERGs recorded from goldfish and cats treated with 6-OHDA were normal, in accord with our finding that the ERG is essentially normal in DA-IPC-depleted zebrafish. These studies all suggest that the outer retina is func- 
tioning reasonably normally in DA-IPC-depleted animals. However, the a- and b-waves of the ERG arise mainly from the photoreceptor, on-bipolar, and Müller cells. Significant alterations in horizontal cell or off-bipolar cell activity induced by DA-IPC depletion are not likely to be obvious in ERG recordings.

\section{Dopamine and inner plexiform layer function}

A main conclusion of our study is that dopamine depletion in the retina has profound effects in the inner plexiform layer, particularly on rod pathways. Cone system function also appears to be compromised in DA-IPC-depleted retinas (Fig. 2A). Although cone function seems to be normal during the first $6-8$ min of dark adaptation, it loses $\sim 1 \log$ unit of sensitivity over the next $6-8$ min of dark adaptation. This suggests that the light-adapted cone system in DA-IPC-depleted animals is normal but that the darkadapted cone system is somewhat abnormal. This conclusion was confirmed in the incremental sensitivity studies; after exposure to bright backgrounds, the sensitivity of DA-IPC-depleted fish is identical to that of control fish (Fig. $3 A$ ).

How might dopamine depletion suppress rod signals in the inner plexiform layer of the retina? In mammals, the rod signal is carried into the inner plexiform layer by bipolar cells that connect exclusively to rods (Polyak, 1941; Boycott and Dowling, 1969). The rod signal is transmitted to cone bipolar cells via the AII amacrine cells, which receive substantial dopaminergic input (Kolb and Famiglietti, 1974; Famiglietti and Kolb, 1975; Dacheux and Raviola, 1986). One could imagine that for the rod signal to be transmitted from the rod to cone bipolar cells in the inner plexiform layer, some dopamine is required. Although it is generally agreed that light enhances dopamine release in the retina, there is substantial evidence that dopamine is also released in darkness (Mangel and Dowling, 1985; Yang et al., 1988a,b; Weiler et al., 1997; Xin and Bloomfield, 1999).

In cold-blooded vertebrates such as fish, there are no known bipolar cells that are rod specific. All bipolar cells that connect to rods also receive some cone input (Stell, 1967; Scholes, 1975). Furthermore, it is believed that these bipolar cells innervate ganglion cells directly, providing the ganglion cells with both rod and cone signals. It is thus difficult to hypothesize how rod signals could be selectively blocked in the inner plexiform layer of coldblooded vertebrates such as zebrafish. However, we have observed in zebrafish that an extensive plexus of DA-IPC processes coexists in the lower part of the inner plexiform layer with the large rod-dominated bipolar cell terminals [E. A. Schmitt and J. E. Dowling, unpublished observations; see also Yazulla and Studholme (1997)]. Thus, the terminals of the large, roddominated bipolar cells in zebrafish would appear to have ready access to, or even be bathed in, dopamine.

\section{Dopamine and retinal circadian rhythmicity}

We previously reported that the absolute visual sensitivity of zebrafish measured behaviorally varies by $\sim 2 \log$ units between early morning and late afternoon hours ( $\mathrm{Li}$ and Dowling, 1998). This rhythmicity of visual sensitivity persists in animals kept in continuous darkness, showing that the rhythm is endogenous. A rhythm in ERG sensitivity to light was also demonstrated, but the extent of threshold variation was only $\sim 1.0 \log$ unit, suggesting that regulation of visual sensitivity by the circadian clock mechanism occurs at several different levels in the visual system $(\mathrm{Li}$ and Dowling, 1998). Several studies have suggested that dopamine is a circadian clock modulator in the retina (Besharse and
Iuvone, 1983; Kolbinger et al., 1990; McCormack and Burnside, 1992; Manglapus et al., 1999). We measured behaviorally as well as by ERG the visual sensitivity of DA-IPC-depleted animals as a function of time of day. No evidence of circadian control of behavioral or ERG sensitivity was detected in DA-IPC-depleted animals, unlike in control animals. The ERG sensitivity of DAIPC-depleted animals was at all times close to the sensitivity of control animals in the late afternoon hours, when the ERG is maximally sensitive (Fig. 6). This suggests that the circadian mechanism acts by decreasing visual sensitivity [see also Li and Dowling (1998)]; that is, visual sensitivity is maximal in the absence of the clock. It may be the case that not all circadian effects are absent in DA-IPC-depleted animals. For example, an OFF-response is observed in the dark-adapted ERG of control fish at subjective dust but not at subjective dawn (Fig. 7). In DA-IPC-depleted fish, an ERG OFF-response is observed at subjective dusk but not at subjective dawn, like the control fish (Fig. 7).

\section{Retinal dopaminergic mechanisms and $n \boldsymbol{n b} b^{+/-}$mutant fish}

The rationale for the experiments described in this paper was to test the hypothesis that the visual deficits exhibited by $n b b^{+/-}$ mutants are related to a malfunction of the dopamine system in the retina [accompanying paper ( $\mathrm{Li}$ and Dowling, 2000)]. In many ways, depletion of the DA-IPCs in wild-type zebrafish mimics the visual defects of $n b b^{+/-}$mutants. In both situations, the visual defect is primarily of the rod system. However, in the $n b b^{+/-}$ mutants, rod system activity measured behaviorally fluctuates dramatically over time, whereas in DA-I PC-depleted animals, the rod system appears completely knocked out. We have recently found that in $n b b^{+/-}$mutants in which the DA-IPCs have been depleted, the behavioral visual thresholds are constant throughout the day and night and identical to those of DA-IPC-depleted wild-type zebrafish (Fig. 5).

In both situations, light sensitizes the retina. That is, the retina appears more normal in the early phases of dark adaptation or after exposure to bright background illumination. In $n b b^{+/-}$fish, light sensitizes both the rods and cones. Quite normal rod and cone function is measured for the first 30 min of dark adaptation; thereafter, visual thresholds rise and begin to fluctuate. In DAIPC-depleted fish, visual thresholds are normal only for the first 6-8 min of dark adaptation, during the cone component of dark adaptation. Thereafter, the visual thresholds rise by $\sim 1.0 \log$ unit and then stabilize. No evidence of any rod system function is seen during any time of dark adaptation. The ERGs of both $n b b^{+/-}$ and DA-IPC-depleted fish are quite normal. On the other hand, ganglion cell thresholds are abnormal in both cases, suggesting that the defects exhibited by both types of animal are manifest in the inner retina.

A possible explanation for the differences between $n b b^{+/-}$and DA-IPC-depleted fish is that DA-IPC-depleted animals represent the extreme case of the $n b b^{+/-}$defect. In the $n b b^{+/-}$ mutation, although there is a defect in DA-IPCs, these cells do function to some extent. Indeed, they may act normally on occasion, explaining the fact that during dark adaptation, the visual thresholds of $n b b^{+/-}$and wild-type fish may be indistinguishable at times. In DA-IPC-depleted animals, on the other hand, the DA-IPCs are completely knocked out, giving rise to the more severe invariant phenotype. That is, the visual sensitivity is always raised, and no threshold fluctuations are seen. 


\section{Regeneration of DA-IPCs}

A final comment concerns the fact that visual threshold recovery occurs in 6-OHDA-treated retinas well before there is complete regeneration of the DA-IPCs. Indeed, the dark adaptation curve of 6-OHDA-treated animals returns to normal after just 16 weeks of recovery, whereas even after 40 weeks after 6-OHDA treatment the number of DA-IPC cells in the retina is only half the normal number [see also Yazulla and Studholme (1997)]. A conclusion that can be drawn from these observations is that a full complement of DA-IPCs is not needed for the maintenance of rod system function in the retina. Several reasons for this may be advanced. For example, the DA-IPCs that have regenerated in the retina may extend their processes all over the retina, or dopamine diffusing throughout the retina from the regenerated DA-IPCs may be sufficient to maintain normal rod and cone visual sensitivity.

\section{Conclusions}

The main conclusion of this study is that dopamine appears to be required for the transmission of rod signals through the inner plexiform layer. In DA-IPC-depleted animals, no rod system activity can be detected behaviorally, but outer plexiform layer function appears relatively normal in these animals, as determined by ERG recordings. However, circadian regulation of ERG light sensitivity is not observed in DA-IPC-depleted animals. Because circadian mechanisms serve to decrease ERG sensitivity (Li and Dowling, 1998), our findings suggest that the outer retina is most sensitive to light in the absence of dopamine, the opposite to that found in the inner retina where dopamine appears to be required for the transmission of rod signals through the inner plexiform layer. How might one reconcile these apparently discrepant results? At the present time, we do not have a simple answer. It is worth noting that dopamine has a multiplicity of effects on neurons throughout the retina, and our findings that dopamine has quite different effects in the outer versus inner retina may reflect this diversity of dopamine actions.

\section{REFERENCES}

Bauer B, Ehinger B, Aberg L (1980) 3H-dopamine release from the rabbit retina. Albrecht Von Graefe's Arch Klin Exp Ophthalmol 215:71-78.

Besharse JC, Iuvone PM (1983) Circadian clock in Xenopus eye controlling retinal serotonin N-acetyltransferase. Nature 305:133-135.

Bodis-Wollner I (1990) Visual deficit related to dopamine deficiency in experimental animals and Parkinson's disease patients. Trends Neurosci 13:296-302.

Bodis-Wollner I, Mitra S, Bobak P, Guillory S, Mylin L (1984) Low frequency distortion in spatio-temporal threshold surface in Parkinson's disease. Invest Ophthalmol Vis Sci [Suppl] 25:313.

Bodis-Wollner I, Marx MS, Mitra S, Bobak P, Yahr M (1987) Visual dysfunction in Parkinson's disease. Brain 110:1675-1698.

Boycott BB, Dowling JE (1969) Organization of the primate retina: light microscopy. Philos Trans R Soc Lond B Biol Sci 225:109-184.

Dacheux RF, Raviola E (1986) The rod pathway in the rabbit retina. A depolarizing bipolar and amacrine cell. J Neurosci 6:331-345.

Dearry A, Burnside B (1989) Light-induced dopamine release from teleost retinas acts as a light-adaptive signal to the retinal pigment epithelium. J Neurochem 53:870-878.

Dowling JE (1987) The retina: an approachable part of the brain. Cambridge, MA: Harvard UP.

Dowling JE (1991) Retinal neuromodulation: the role of dopamine. Vis Neurosci 7:87-97.

Dowling JE, Ehinger B (1975) Synaptic organization of the aminocontaining interplexiform cells of the goldfish and Cebus monkey retina. Science 188:270-273.

Dowling JE, Ehinger B (1978) The interplexiform cell system. I. Syn- apses of the dopaminergic neurons of the goldfish retina. Proc R Soc Lond B Biol Sci 201:7-26.

Fadool JM, Fadool DA, Moore JC, Linser PJ (1999) Characterization of monoclonal antibodies against zebrafish retina. Invest Ophthal Vis Sci 40:S237.

Famiglietti V, Kolb H (1975) A bistratified amacrine cell and synaptic circuitry in the inner plexiform layer of the retina. Brain Res 84:293-300.

Godley BF, Wurtman RJ (1988) Release of endogenous dopamine from the superfused rabbit retina in vitro: effect of light stimulation. Brain Res 452:393-395.

Hampson EC, Vaney DI, Weiler R (1992) Dopaminergic modulation of gap junction permeability between amacrine cells in mammalian retina. J Neurosci 12:4911-4922.

Jensen RJ, Daw NW (1984) Effects of dopamine antagonists on receptive fields of brisk cells and directionally selective cells in the rabbit retina. J Neurosci 4:2972-2985.

Jensen RJ, Daw NW (1986) Effects of dopamine and its agonists and antagonists on the receptive fields properties of ganglion cells in the rabbit retina. Neuroscience 17:837-855.

Kirsch M, Wagner HJ (1989) Release pattern of endogenous dopamine in teleost retinae during light adaptation and pharmacological stimulation. Vision Res 29:147-154.

Knapp AG, Schmidt KF, Dowling JE (1990) Dopamine modulates the kinetics of ion channels gated by excitatory amino acids in retinal horizontal cells. Proc Natl Acad Sci USA 87:767-771.

Kolb H, Famiglietti V (1974) Rod and cone pathway in the inner plexiform layer of cat retina. Science 186:47-49.

Kolbinger W, Kohler K, Oetting H, Weiler R (1990) Endogenous dopamine and cyclic events in the fish retina. I: HPLC assay of total content, release, and metabolic turnover during different light/dark cycles. Vis Neurosci 5:143-149.

Kramer SG (1971) Dopamine: a retinal neurotransmitter. I. Retinal uptake, storage and light-stimulated release of $3 \mathrm{H}$-dopamine in vivo. Invest Ophthalmol 10:438-452.

Krizaj D, Gabriel R, Owen WG, Witkovsky P (1998) Dopamine $\mathrm{D}_{2}$ receptor-mediated modulation of rod-cone coupling in the Xenopus retina. J Comp Neurol 398:529-538.

Li L, Dowling JE (1997) A dominant form of inherited retinal degeneration caused by a non-photoreceptor cell-specific mutation. Proc Natl Acad Sci USA 94:11645-11650.

Li L, Dowling JE (1998) Zebrafish visual sensitivity is regulated by a circadian clock. Vis Neurosci 15:851-857.

Li L, Dowling JE (2000) Disruption of the olfactoretinal centrifugal pathway may relate to the visual system defect in night blindness $b$ mutant zebrafish. J Neurosci 20:1883-1892.

Lin ZS, Yazulla S (1994a) Depletion of retinal dopamine increases brightness perception in goldfish. Vis Neurosci 11:683-693.

Lin ZS, Yazulla S (1994b) Depletion of retinal dopamine does not affect the ERG b-wave increment threshold function in goldfish in vivo. Vis Neurosci 11:695-702.

Liu Y, Lasater EM (1994) Calcium currents in turtle retinal ganglion cells II. Dopamine modulation via a cyclic AMP-dependent mechanism. J Neurophysiol 71:743-752.

Maguire G, Hamasaki DI (1994) The retinal dopamine network alters the adaptational properties of retinal ganglion cells in the cat. J Neurophysiol 72:730-740.

Maguire G, Smith EL (1985) Cat retinal ganglion cell receptive-field alterations after 6-hydroxydopamine induced dopaminergic amacrine cell lesions. J Neurophysiol 53:1431-1443.

Maguire G, Werblin F (1994) Dopamine enhances a glutamate-gated ionic current in OFF bipolar cells of the tiger salamander retina. J Neurosci 14:6094-6101.

Mangel SC, Dowling JE (1985) Responsiveness and receptive field size of carp horizontal cells are reduced by prolonged darkness and dopamine. Science 229:1107-1109.

Manglapus MK, Iuvone PM, Underwood H, Pierce ME, Barlow RB (1999) Dopamine mediates circadian rhythms of rod-cone dominance in the Japanese quail retina. J Neurosci 19:4132-4141.

Masson G, Mestre D, Blin O (1993) Dopaminergic modulation of visual sensitivity in man. Fundam Clin Pharmacol 7:449-463.

McCormack CA, Burnside B (1992) A role for endogenous dopamine in circadian regulation of retinal cone movement. Exp Eye Res 55:511-520.

Miller R, Horn A, Iversen L (1974) Effects of dopamine-like drugs on rat 
striatal adenyl cyclase have implications for CNS dopamine receptor topography. Nature 250:238-241.

Negishi K, Teranishi T, Kato S (1982a) New dopaminergic and indoleamine-accumulating cells in the growth zone of goldfish retinas after neurotoxic destruction. Science 216:747-749.

Negishi K, Teranishi T, Kato S (1982b) Growth zone of the juvenile goldfish retina revealed by fluorescent flat mounts. J Neurosci Res 7:321-330.

Nicola SM, Kombian SB, Malenka RC (1996) Psychostimulants depress excitatory synaptic transmission in the nucleus accumbens via presynaptic D1-like dopamine receptors. J Neurosci 16:1591-1604.

Polyak SL (1941) The retina. Chicago: University of Chicago.

Schmitt EA, Dowling JE (1996) Comparison of topographical patterns of ganglion and photoreceptor cell differentiation in the retina of the zebrafish. J Comp Neurol 371:222-234.

Scholes JH (1975) Colour receptors and their synaptic connexions in the retina of a cyprinid fish. Philos Trans R Soc Lond B Biol Sci 270:61-118.

Stell WK (1967) The structure and relationship of horizontal cells and photoreceptor-bipolar synaptic complexes in goldfish retina. Am J Anat 121:401-424.

Teranishi T, Negishi K, Kato S (1983) Dopamine modulation of S-potential amplitude and dye-coupling between external horizontal cells in carp retina. Nature 301:243-246.

Umino O, Dowling JE (1991) Effects of light stimuli on the release of dopamine from interplexiform cells in the white perch retinas. Vis Neurosci 7:451-458.

Voigt T, Wassle H (1987) Dopaminergic innervation of AII amacrine cells in mammalian retina. J Neurosci 7:4115-4128.

Wagner HJ, Behrens UD, Zaunreiter M, Douglas RH (1992) The circadian component of spinule dynamics in teleost retinal horizontal cells is dependent on the dopaminergic system. Vis Neurosci 9:345-351.
Weiler R, Baldridge WH, Mangel SC, Dowling JE (1997) Modulation of endogenous dopamine release in the fish retina by light and prolonged darkness. Vis Neurosci 14:351-356.

Westerfield M (1995) The zebrafish book: a guide for the laboratory use of zebrafish (Brachydanio rerio). Eugene, OR: University of Oregon.

Witkovsky P, Dearry A (1991) Functional roles of dopamine in the vertebrate retina. Prog Retinal Res 11:247-292.

Witkovsky P, Shi SP (1990) Slow light and dark adaptation of horizontal cells in the Xenopus retina: a role for endogenous dopamine. Vis Neurosci 5:405-413.

Witkovsky P, Nicholson C, Rice ME, Bohmaker K, Meller ME (1993) Extracellular dopamine concentration in the retina of the clawed frog, Xenopus laevis. Proc Natl Acad Sci USA 90:5667-5671.

Xin D, Bloomfield SA (1999) Dark- and light-induced changes in coupling between horizontal cells in mammalian retina. J Comp Neurol 405:75-87.

Yang XL, Tornqvist K, Dowling JE (1988a) Modulation of cone horizontal cell activity in the teleost fish retina. I. Effects of prolonged darkness and background illumination on light responsiveness. J Neurosci 8:2259-2268.

Yang XL, Tornqvist K, Dowling JE (1988b) Modulation of cone horizontal cell activity in the teleost fish retina. II. Role of interplexiform cells and dopamine in regulating light responsiveness. J Neurosci 8:2269-2278

Yazulla S, Studholme KM (1997) Differential reinnervation of retinal bipolar cell dendrites and axon terminals by dopamine interplexiform cells following dopamine depletion with 6-OHDA. J Comp Neurol 382:535-545.

Yazulla S, Zucker CL (1988) Synaptic organization of dopaminergic interplexiform cells in the goldfish retina. Vis Neurosci 1:13-30.

Zucker CL, Dowling JE (1987) Centrifugal fibres synapse on dopaminergic interplexiform cells in the teleost retina. Nature 300:166-168. 